Background: Accumulation of amyloid beta in the brain is one of the first events in Alzheimer's disease (AD) and starts decades before symptoms arise. Disruption of resting state functional connectivity (FC) might represent early functional consequences associated with amyloid pathology. This study examines the association of FC for 10 resting state networks (RSNs) with amyloid pathology in a sample of cognitively healthy elderly monozygotic twins. In addition, we estimated the upper limit of genetic contribution to RSN-FC, by examining the within twin pair similarity of this measure. Methods: Monozygotic twins were selected from the EMIF-AD PreclinAD study. Inclusion criteria were age $\geq 60$ years and having a delayed recall score above $-1.5 \mathrm{SD}$ of normative data. FSLMELODIC was used to identify ten large-scale functional brain networks from resting state fMRI data. Amyloid pathology was quantified from $\left[{ }^{18} \mathrm{~F}\right]$ Flutemetamol PET scans. Dual regression analysis was performed to test the association between the RSNs and amyloid pathology. To determine twin correlations in FC for each network of interest, correlation analysis was performed using the subject specific mean FC. Analyses were adjusted for age, sex and grey matter atrophy. Results: We included 57 twin pairs and 13 individual twins (total 127 subjects) with a mean age of 69 years $(\mathrm{SD}=6.97)$. We found that amyloid pathology was positively associated with FC between precuneus /posterior cingulate cortex (PCC) and the sensorimotor network. Within twin-pair correlations for RSN-FC ranged from .27 (auditory) to .49 (visual network). Conclusions: The observed increase in FC between the precuneus/ PCC and the sensorimotor network in subjects with increasing amyloid pathology might reflect the capacity of the brain to compensate for early amyloid pathology. Future research should investigate whether individual differences in functional compensation might be predictive for developing AD. Further, our data suggests that in addition to a moderate genetic background for FC, also non-genetic factors substantially influence this trait. Next step will be to study which non-genetic risk factors are involved. This work has received support from the EU/EFPIA Innovative Medicines Initiative Joint Undertaking EMIF grant agreement $n^{\circ} 115372$.

\section{IC-P-054 EXAMINING LONGITUDINAL NEUROIMAGING PATTERNS IN AUTOSOMAL DOMINANT ALZHEIMER DISEASE: RESULTS FROM THE DOMINANTLY INHERITED ALZHEIMER NETWORK}

Brian A. Gordon ${ }^{1,2}$, Tyler Blazey ${ }^{1}$, Yi Su ${ }^{1}$, Amrita Hari-Raj ${ }^{1}$, Aylin Dincer ${ }^{1}$, Shaney Flores ${ }^{1}$, Jon Christensen ${ }^{1}$, Eric McDade ${ }^{1,2}$, Guoqiao Wang $^{1,2}$, Chengjie Xiong ${ }^{1,2}$, Nigel J. Cairns ${ }^{1,2}$, Jason Hassenstab $^{1,2}$, Anne M. Fagan ${ }^{1,2,3}$, Daniel S. Marcus ${ }^{1}$, Clifford R. Jack Jr. ${ }^{4}$, Robert A. Koeppe ${ }^{5}$, Russ C. Hornbeck ${ }^{1}$, Katrina L. Paumier ${ }^{1,2}$, Beau M. Ances ${ }^{1,2}$, David M. Holtzman ${ }^{1,2,3}$, Marcus E. Raichle ${ }^{1,2,3}$, John C. Morris ${ }^{1,2,3}$, Randall J. Bateman ${ }^{1,2,3}$, Tammie L. S. Benzinger ${ }^{1,2},{ }^{1}$ Washington University School of Medicine, St. Louis, MO, USA ${ }^{2}$ Knight Alzheimer's Disease Research Center, St. Louis, MO, USA; ${ }^{3}$ Hope Center for Neurological Disorders, St. Louis, MO, USA $;{ }^{4}$ Mayo Clinic, Rochester, MN, USA; ${ }^{5}$ University of Michigan, Ann Arbor, MI, USA. Contact e-mail: bagordon@wustl.edu

Background:Models of Alzheimer pathology propose that amyloidosis, hypometabolism, and structural declines emerge not only over time, but also spatially in the brain. Autosomal dominant Alzheimer disease (ADAD) provides an elegant way to study the evolution of such pathology due to the ability to stage an individual relative to their years to expected symptom onset (EYO). Longitu-
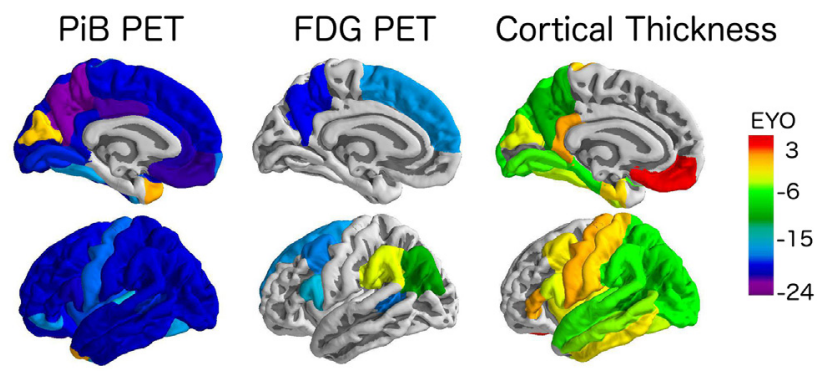

dinal neuroimaging data is a particularly powerful way to model such changes, as within-subject measures reduces between-subject variability caused by unobserved individual differences. Methods: We examined longitudinal PiB, FDG, and structural MRI data in a population of 400 individuals enrolled in the Dominantly Inherited Alzheimer's Network (DIAN). Data were processed examining 34 cortical and 7 subcortical regions. We utilized linear mixed effects models to explore changing pathology. Models were fit for each region included fixed effects for mutation status, time from baseline, baseline EYO, and all possible two and three-way interactions. To allow non-linearities, EYO was modeled as a restricted cubic spline. Analyses were run separately for each modality. The primary focus was on the first time point in the disease where biomarker change in that region differed between mutation carrier and non-carriers. Results: Relative to non-carriers, rates of amyloid deposition were significantly higher in carriers at an average EYO across regions of -18.2, glucose metabolism began declining at an average EYO of -14.2, and MRI structural measures declined at an
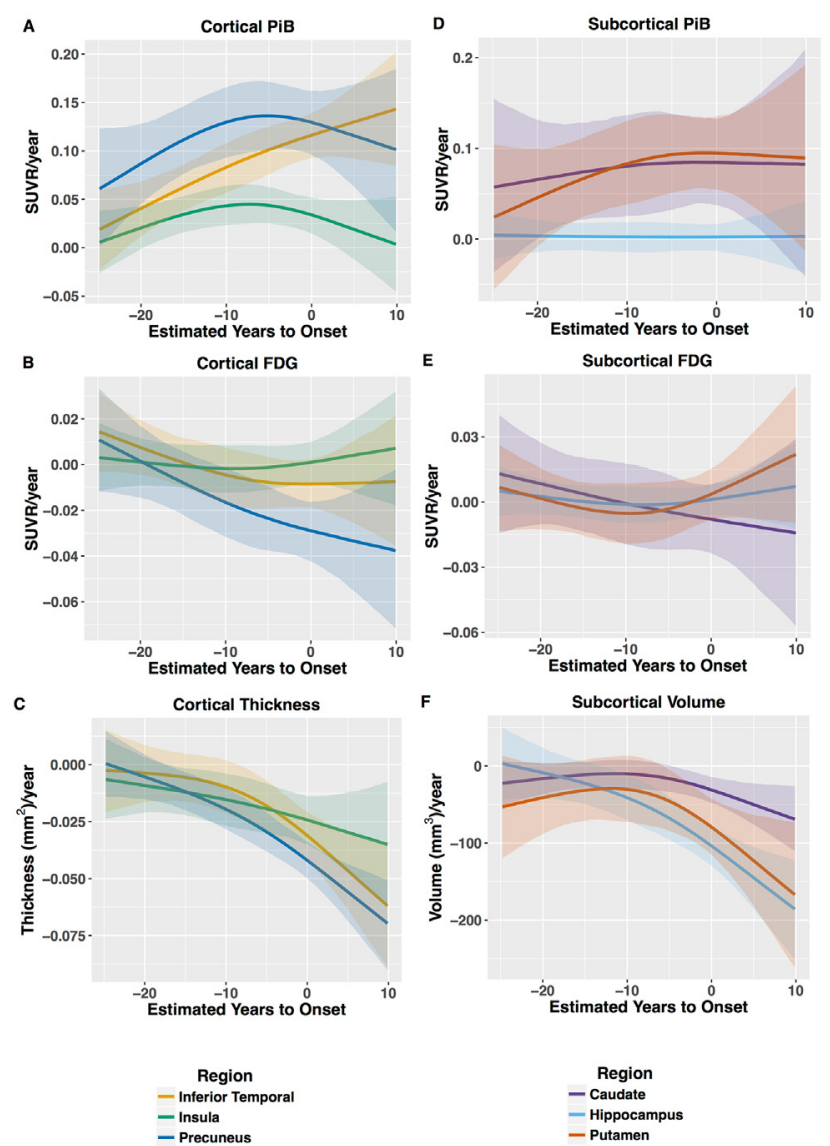
average EYO of -4.2. While this three-stage pattern was common across regions of the brain, it was not ubiquitous (Figure 1). Most prominently a subset of regions demonstrated elevated PiB PET accumulation and structural declines, but not abnormal glucose utilization. Further, the regional timing within a modality (e.g. PiB PET) was highly variable across brain regions (Figure 2). Conclusions: Our study presents both the longitudinal temporal trajectories of and spatial patterns of Alzheimer pathology in ADAD cohorts. Our results are consistent with prior theoretical models and crosssectional work suggesting that initial increases in amyloid accumulation are followed by hypometabolism, and finally by structural atrophy. We demonstrated that there is not one temporal relationship, but that the emergence of pathology varies across the brain.

\section{IC-P-055 EFFECT OF APOE- 22 ON REGIONAL GRAY MATTER ATROPHY AND CLINICAL PHENOTYPE IN ALZHEIMER'S DISEASE}

Colin Groot $^{1,2}$, Chris W. J. van der Weijden ${ }^{3,4}$, Wiesje M. van der Flier $^{5,6}$, Anita C. van Loenhoud ${ }^{2}$, Bart N. M. van Berckel', Frederik Barkhof ${ }^{8,9}$, Teddy Koene ${ }^{10}$, Charlotte E. Teunissen ${ }^{11}$, Philip Scheltens ${ }^{5}$, Rik Ossenkoppele ${ }^{1,5},{ }^{1}$ Department of Radiology and Nuclear Medicine, Amsterdam Neuroscience, VU University Medical Center, Amsterdam, Netherlands; ${ }^{2}$ Alzheimer Center and Department of Neurology, VU University Medical Center, Amsterdam Neuroscience, Amsterdam, Netherlands; ${ }^{3}$ Department of Radiology and Nuclear Medicine, Neuroscience Campus Amsterdam, VU University Medical Center, Amsterdam, Netherlands; ${ }^{4}$ Alzheimer Center, Department of Neurology, Neuroscience Campus Amsterdam, VU University Medical Center, Amsterdam, Netherlands; ${ }^{5}$ Alzheimer Center and Department of Neurology, Amsterdam Neuroscience, VU University Medical Center, Amsterdam, Netherlands; ${ }^{6}$ Department of Epidemiology and Biostatistics, VU University Medical Center, Amsterdam, Netherlands; ${ }^{7}$ Department of Radiology and Nuclear Medicine, Amsterdam Neuroscience, VU University Medical Center, Amsterdam, Netherlands; ${ }^{8}$ Radiology and Nuclear Medicine, VU University Medical Center, Amsterdam, Netherlands; ${ }^{9}$ Institutes of Neurology and Healthcare Engineering, UCL, London, United Kingdom; ${ }^{10}$ Department of Medical Psychology, Neuroscience Campus Amsterdam, VU University Medical Center, Amsterdam, Netherlands, ${ }^{11}$ Department of Biochemistry, VU University Medical Center, Amsterdam Neuroscience, Amsterdam, Netherlands. Contact e-mail: c.groot3@vumc.nl

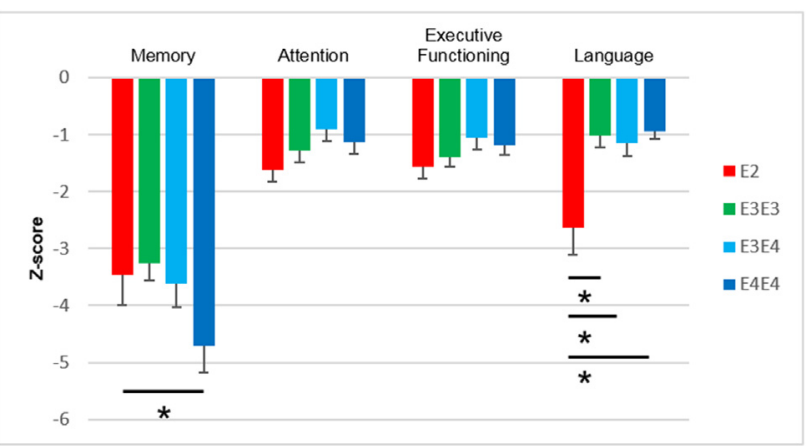

Figure 1. Neuropsychological performance of all amyloid-positive groups across cognitive domains

Z-scores, calculated based on the mean and standard deviation of the normal control group indicate better memory performance of $\varepsilon 2$ compared to $\varepsilon 4 / \varepsilon 4$ and worse language performance of $\varepsilon 2$ compared to all groups.

* significant contrast with $\varepsilon 2$ carriers at $\alpha=.05$, adjusted for age, sex, education and disease stage.

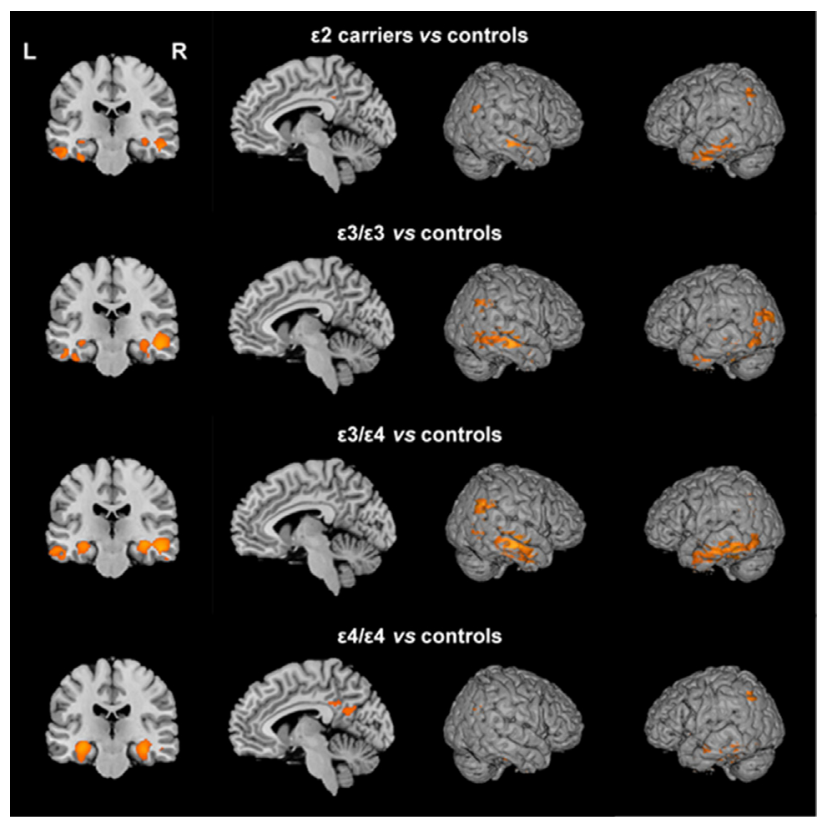

Figure 2. Voxelwise contrasts between normal controls and amyloid-beta positive $\varepsilon 2, \varepsilon 3 / \varepsilon 3, \varepsilon 3 / \varepsilon 4$ and $\varepsilon 4 / \varepsilon 4 \mathrm{AD}$ patients, family-wise error corrected $(\mathrm{p}<0.05)$

Colored areas indicate regions where $\mathrm{AD}$ groups have significantly lower gray matter volume than normal controls.

Background: The APOE $\varepsilon 2$ allele is considered a protective factor against developing Alzheimer's disease (AD). Consequently, the proportion of APOE $\varepsilon 2$ carriers among AD patients is low (3-5\%), but a small number of individuals do develop AD despite $\varepsilon 2$ carriership. In this study, we characterized the neuropsychological profile

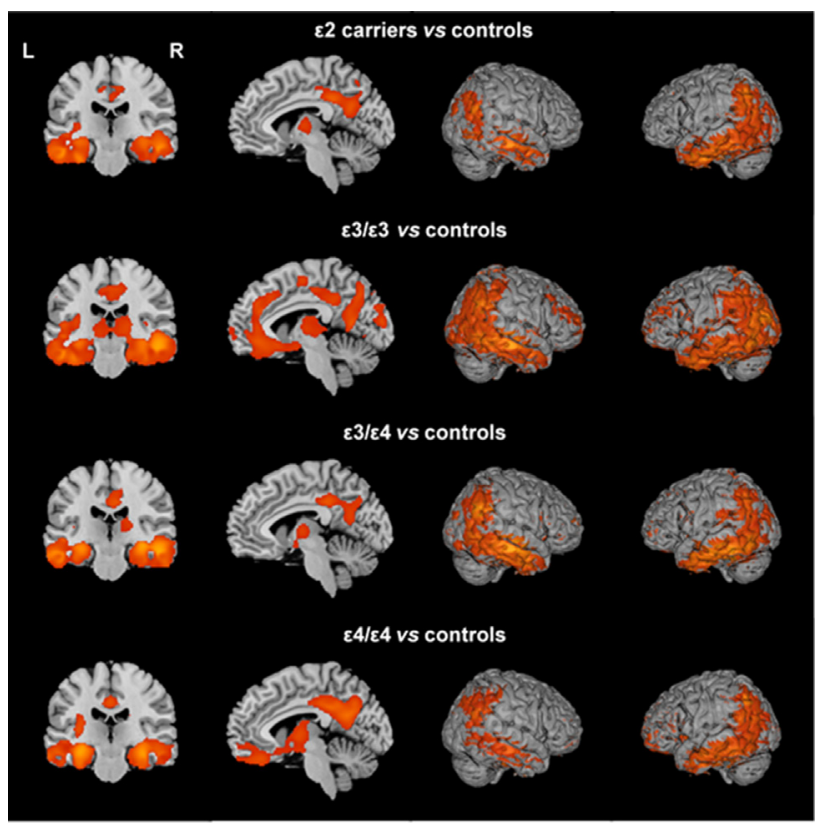

Figure 3. Voxelwise contrasts between normal controls and amyloid-beta positive $\varepsilon 2, \varepsilon 3 / \varepsilon 3, \varepsilon 3 / \varepsilon 4$ and $\varepsilon 4 / \varepsilon 4 \mathrm{AD}$ patients, $(\mathrm{p}<0.001$, uncorrected for multiple comparisons)

Colored areas indicate regions where $\mathrm{AD}$ groups have significantly lower gray matter volume than normal controls. 